\title{
Sandwich-structured bamboo powder/glass fibre-reinforced epoxy hybrid composites - mechanical performance in static and dynamic evaluations
}

\begin{abstract}
A layer of woven E-glass fibre was embedded each at top and bottom layer of bamboo-filled epoxy, producing sandwich-structured hybrid composites. The incorporation of woven glass fibre was intended to slow down the crack propagation in composites. Different bamboo powder loading was applied to analyse its effect on the static and dynamic mechanical properties of composites. The composite with the lowest bamboo powder loading of $10 \%$ marked the highest tensile strength, where the strength decreased as the loading increased. Poor bamboo-epoxy adhesion, observed from the SEM, leads to more chances of pull out fibres when tensile load was applied. Opposite trend was observed in flexural strength, where the highest loading of $30 \%$ marked the highest value of flexural strength. The dynamic evaluation was carried out with respect to temperature, which ranged from $25^{\circ} \mathrm{C}$ to $150^{\circ} \mathrm{C}$, and at a fixed frequency of $1 \mathrm{~Hz}$. Effective stress transfer between the fibre and the matrix takes place in composites with 30\% bamboo powder loading, shown by the lowest peak height of the Tan $\delta$ curves. The significant increase in the storage modulus values for the composites at low temperature compared to the rubbery plateau region at high temperature suggested the high utility of the composites at low temperature regardless of the effect of the percentage increment in bamboo powder loading. These results showed that the bamboo powder inclusion helps in bestowing stiffness to the composites with $30 \%$ as the optimum percentage in this dynamic mechanical evaluation. It can be suggested that the introduction of woven-typed fibre into short fibre composites in sandwich structure, as discussed in this study, can slow down the failure of composites, and thus notify users before the total failure.
\end{abstract}

Keyword: Composites; Dynamic mechanical analysis; Bamboo composites; Glass fibre; Modulus 